\title{
A CROSS-COUNTRY ANALYSIS ON CAPITAL FLOWS CYCLE: STYLIZED FACTS AND REGIONAL SYNCHRONIZATION
}

\section{Yang Yang ${ }^{1}$ \\ Haizhen Yang ${ }^{2+}$ \\ Mengting Zhang ${ }^{3}$}

\author{
${ }^{1,2, s}$ School of Economics and Management, University of Chinese Academy of \\ Sciences, Beijing, China. \\ Email: yansyang185@mails.ucas.ac.cn Tel: +8618811528398 \\ 'Email: haizheny@ucas.ac.cn Tel +8613661397716 \\ 'Email: zhangmt43@163.com Tel +8618813031602
}

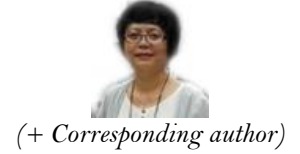

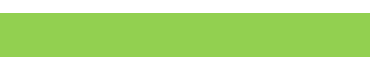

Article History

Received: 2 February 2021 Revised: 4 March 2021

Accepted: 29 March 2021

Published: 23 April 2021

\section{Keywords}

Capital flows

Cyclical characteristics

Regional synchronization

Advanced economies

Emerging and developing

economies

BBQ cycle algorithm.

JEL Classification:

F39.

Contribution/Originality: This study contributes to the existing literature that investigates the characteristics and the regional synchronization of capital flows cycle and is one of very few studies which have conducted a systematic cross-country analysis of the cyclical characteristics of capital flows.

\section{INTRODUCTION}

Capital flows often come in waves and have strong cyclical profiles, as previous literature has documented (Broner, Didier, Erce, \& Schmukler, 2013; Forbes \& Warnock, 2012; Reinhardt \& Dell'Erba, 2013). Many countries have experienced waves of capital flows over the past three decades. More specifically, for a certain country, capital inflows increase during economic expansions and contract substantially during crises and recessions. Such a profile may trigger currency devaluations, asset price bubble bursts and economic slowdowns (Burger \& Ianchovichina, 2014; Calderón \& Kubota, 2014; Dagher \& Jihad, 2010). Therefore, to understand and regulate the abnormal fluctuations of capital flows, an in-depth analysis on the characteristics of capital flows is needed.

There is a wide range of literature concerning the characteristics of capital flows. One strand of literature focuses on the pro- or counter-cyclical behavior of capital flows (Broner et al., 2013; Davis, 2015; Kim \& Zhang, 2020). Another strand of literature discusses the volatility of capital flows during various periods or among different capital flow types (Broner \& Rigobon, 2004; Broto, Díaz-Cassou, \& Erce, 2011; Neumann, Penl, \& Tanku, 2009). In 
recent years, the characteristics of extreme volatility of international capital flows has increasingly emerged. Therefore, some scholars shift towards the theme of extreme capital flows (Calvo, 1998; Cowan, De Gregorio, Micco, \& Neilson, 2008; Forbes \& Warnock, 2012; Forbes \& Warnock, 2020; Reinhart \& Reinhart, 2008; Rothenberg \& Warnock, 2011). However, there are few scholars conducting systematic cross-country analysis on the cyclical characteristics of capital flows. Park (2018) initiatively used the business cycle approach to investigate the cyclical characteristics of capital flows and further discusses whether national and regional heterogeneity for capital flows cycles exists. However, this research is limited to gross capital inflows in emerging economies; there is no discussion on gross capital outflows and net capital flows. In addition, as the major source of capital inflows to emerging economies, advanced economies are not taken into consideration. In summary, there are still large research gaps regarding the theme of capital flows cycle. For example, how does the capital flows cycle behave for different countries and regions? Have the characteristics of capital flows cycles changed since the global financial crisis in 2008? Is there a regional synchronization of capital flows cycles? Are there obvious differences in the cyclical characteristics of different types of capital flows? Addressing these questions will not only be conducive to thoroughly understanding the characteristics of capital flows, but also provide references for management of capital flow risks.

In order to answer the above questions, we extended the study by Park (2018). Specifically, this paper covers not only gross capital inflows, but also net capital flows, gross capital outflows and different types of capital flows (net direct investment, net portfolio investment and net other investment). In addition, we also took advanced economies into consideration. This study was carried out from the following three aspects. First, we identified the capital flows (net and gross) cycles of 32 advanced economies and 28 emerging and developing economies over the period from Q1 1980 to Q1 2020 using the BBQ algorithm to explore the features of the capital flows cycles from the perspectives of duration, amplitude and speed. The results show that small economies generally have higher amplitude and faster speed of net capital flows compared with large economies; moreover, the speed of portfolio investment and other investment is significantly faster than that of direct investment in most developed, emerging and developing countries. Furthermore, by identifying the regional net capital flows cycles, we found that the duration of capital flows cycle had shortened significantly in most regions after the global financial crisis in 2008. Finally, the paper investigates the regional synchronization of capital flows cycles, and the results show that the degree of regional synchronization of capital flows cycles within advanced economies is high, while that within emerging and developing economies is relatively low. In addition, capital flows cycles between advanced Asian economies and any region of emerging and developing economies is highly synchronized.

The remainder of the paper is as follows. Section 2 introduces the identification method of international capital flows (net and gross) cycles and the data used in this paper; Section 3 investigates the cyclical characteristics of capital flows for individual economies as well as different regions; Section 4 explores the synchronization of capital flows cycles across regions; and Section 5 summarizes the results of the analysis.

\section{METHOD AND DATA}

According to Bluedorn, Duttagupta, Guajardo, \& Topalova (2013), capital inflows of both advanced and emerging economies have expanded significantly from the mid-1990s to the first half of the 2000s. Subsequently, they declined sharply during the global financial crisis in 2008. By 2009, the volume of capital inflows to those economies began to rebound and then fell again in 2011 accompanied by the European sovereign debt crisis. In summary, past experiences demonstrate that capital flows have an obvious cyclical profile of alternating expansions and contractions, and generally exhibit greater volatility accompanied by crises.

Taking the above stylized facts of capital flows into consideration, first, we defined the capital flows cycle as the alternating movement of the expansion and contraction of capital flows. As illustrated in Figure 1, from the trough of capital flows to the neighboring peak is the expansion period, and from the peak to the next trough is a 
contraction period. The amplitude represents the distance from the peak to the trough. More specifically, the amplitude of the expansion phase is the distance from the trough to the peak, and that of the contraction phase is the distance from the peak to the next trough. In addition, to eliminate the effect of the distance symbol, we take the absolute value. A higher amplitude means more volatile capital flows. Finally, the speed is calculated as the amplitude divided by the duration. Moreover, we separately divided the cycles for net capital flows, gross capital inflows, gross capital outflows, and different types of capital flows (direct investment, portfolio investment and other investment).

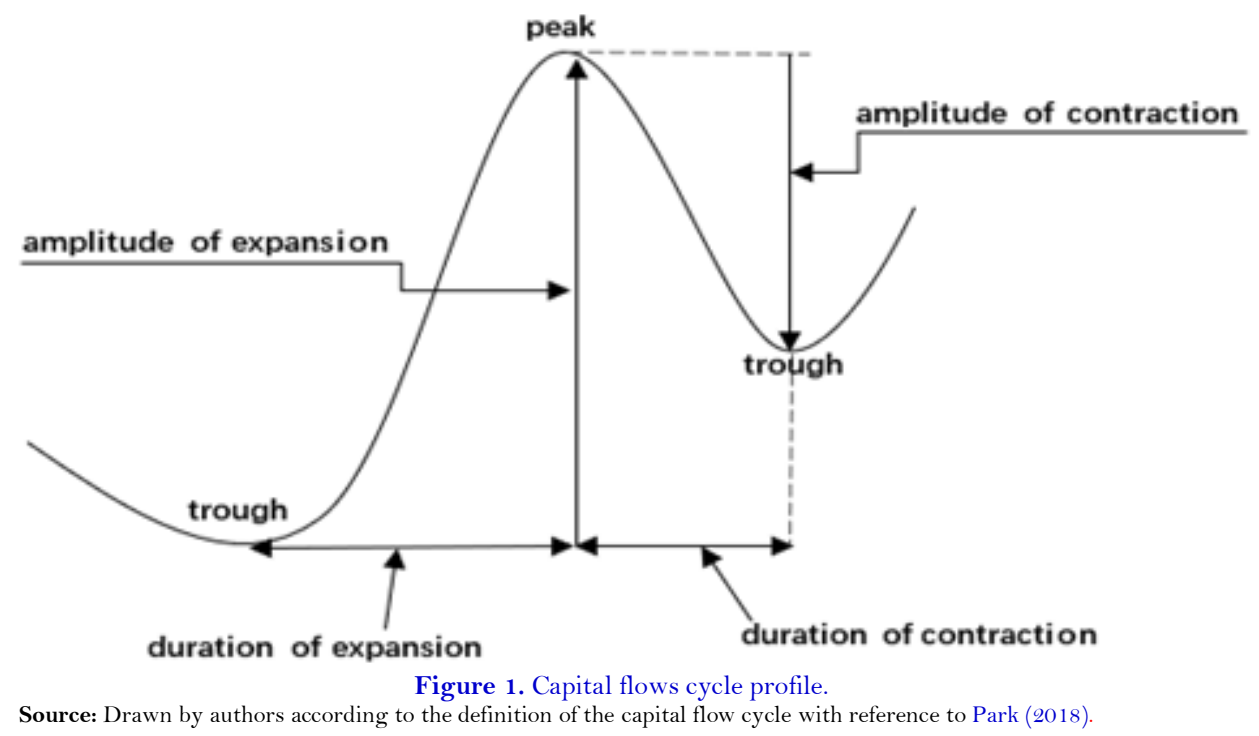

Regarding the method of dividing the capital flows cycle, we used the BBQ algorithm (Harding \& Pagan, 2002), an algorithm widely applied to business cycles. The three steps of the BBQ algorithm are as follows:

Step 1: Identify possible turning points (peaks and troughs) of capital flows series in an individual economy, where the series $\left\{y_{t}\right\}$ represents local extreme points at moment t; the peaks and troughs should satisfy the following conditions: $y_{t}>y_{t \pm i}, 1 \leq i \leq k ; y_{t}<y_{t \pm i}, 1 \leq i \leq k$; where $\mathrm{k}=2$ for quarterly data.

Step 2: Eliminate consecutive peaks and troughs to ensure that they both alternate. If there are several consecutive peaks (troughs), choose the highest peaks (the lowest troughs). If there are several identical peaks (troughs) in a row, choose the last peak (trough) as the turning point.

Step 3: Based on the duration principles, the duration of trough-to-peak or peak-to-trough is at least two quarters, and the duration of the whole cycle is at least five quarters. Accordingly, we removed some turning points that did not satisfy the requirements.

Our capital flows data are from the IMF Balance of Payments Statistics ${ }^{1}$, spanning 60 economies (32 advanced economies and 28 emerging and developing economies)2, from Q1 1980 to Q1 2020. The capital flows data have been normalized (capital flow scale divided by nominal GDP).

\footnotetext{
${ }^{1}$ see https://data.imf.org/?sk=4c5 14d48-b6ba-49ed-8ab9-52b0c 1 a0179b for details.

2 Advanced economies include Asia: Hong Kong, China, Israel, Japan, Korea, Singapore; Europe: Austria, Belgium, the Czech Republic, Denmark, Estonia, Finland, F rance, Germany, Greece, Iceland, Ireland, Italy, Latvia, Lithuania, the Netherlands, Norway, Portugal, Slovakia, Slovenia, Spain, Sweden, Switzerland, United Kingdo m; North America: Canada, United States; Oceania: Australia, New Zealand. Emerging and developing economies include Asia: Armenia, China, Georgia, India, Indo nesia, Kazakhstan, Malaysia, Mongolia, Philippines, Thailand, Vietnam; Europe: Belarus, Bulgaria, Croatia, Hungary, Moldova, Poland, Romania, Russia, Turkey, Uk raine; North America: Mexico; South America: Argentina, Brazil, Chile, Colombia, Ecuador; Africa: South Africa.
} 


\section{CYCLICAL CHARACTERISTICS OF CAPITAL FLOWS IN INDIVIDUAL COUNTRIES AND DIFFERENT REGIONS}

3.1 Cyclical Characteristics of Capital Flows in Individual Countries

3.1.1 Duration

The duration of the capital flows cycle partly reflects the volatility of capital flows; a shorter duration means more violent fluctuation. Overall, the average duration of the net capital flows cycle is generally shorter than that of gross capital flows. This can be attributed to the fact that net capital flows are derived from gross capital inflows minus gross capital outflows, and the difference of the series exhibits greater volatility.

Regarding advanced economies (see Table 1), some large economies with a mature financial system have a longer net capital flows cycle, such as France (17.25 quarters), the United States (16.89 quarters), Japan (16.50 quarters) and Canada (16.28 quarters). Moreover, contraction periods are generally longer than the expansion periods in these economies. By contrast, the duration of the net capital flows cycle is relatively shorter in small developed economies with a strong financial system, such as Hong Kong, China (7.05 quarters), Singapore (10.11 quarters) and New Zealand (8.69 quarters). However, unlike most advanced economies, these economies' contraction periods are generally shorter than their expansion periods. Looking at different types of capital flows, the duration of direct investment is generally longer than that of portfolio investment and other investment in Asian countries. Specifically, the duration of direct investment in Singapore (19 quarters) is the longest, followed by Hong Kong, China (14.07 quarters) and Korea (12.49 quarters). This result is consistent with the long-term feature of direct investment. However, the situation in most European and North American economies is reversed; the duration of other investment and portfolio investment is longer than that of direct investment. Regarding other investment, the duration of the cycle in Canada (21.93 quarters) is the longest among major advanced countries. The top three economies with the longest portfolio investment cycle are the United States (15.30 quarters), the United Kingdom (14.87 quarters) and Germany (14.15 quarters). The results indicate that these countries attract large-scale short-term capital by virtue of their developed financial markets and economic strength.

In emerging and developing economies (see Table 2), contraction periods of capital flows are generally shorter than expansion periods, especially in Bulgaria, Malaysia, Thailand and Argentina. Therefore, these countries should pay more attention to preventing the risk of sudden stops of capital flows. Among the three types of capital flows, there is no significant difference in average duration of capital flows cycle (direct investment: 12.39 quarters, portfolio investment: 12.81 quarters, other investment: 13.48 quarters). For direct investment, the duration of cycles are relatively long in some major emerging economies, such as Bulgaria (18 quarters), Malaysia (15 quarters) and China (14.83 quarters). For portfolio investment, the average duration of cycles in the European region (14.06 quarters) is significantly longer than in other regions, which may be due to Europe having a relatively mature securities market. For other investment, the average duration in the South American region (19.19 quarters) is significantly longer than other regions, and Chile (47.50 quarters) pulls up the average value for the region. 
Table 1. Duration of capital flows in advanced economies (unit: quarter).

\begin{tabular}{|c|c|c|c|c|c|c|c|c|c|c|c|c|c|c|c|c|c|c|}
\hline \multirow[b]{2}{*}{ AD Economies } & \multicolumn{3}{|c|}{ Net Flows } & \multicolumn{3}{|c|}{ Gross Inflows } & \multicolumn{3}{|c|}{ Gross Outflows } & \multicolumn{3}{|c|}{ Net DI } & \multicolumn{3}{|c|}{ Net PI } & \multicolumn{3}{|c|}{ Net OI } \\
\hline & $(1)$ & $(2)$ & $(3)$ & 1) & $(2)$ & $(3)$ & $(1)$ & $(2)$ & $(3)$ & $(1)$ & $(2)$ & $(3)$ & $(1)$ & $(2)$ & $(3)$ & $(1)$ & $(2)$ & $(3)$ \\
\hline 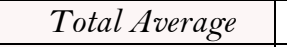 & 99 & 4 & .53 & 1.00 & 91 & 33 & 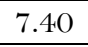 & 4 & 14.65 & 6.36 & 6 & 12.72 & 43 & 6.23 & 12.66 & 6.37 & 6.71 & 13.08 \\
\hline & .74 & 08 & 0.81 & 7.62 & .56 & 13. & 7.61 & 20 & 14.81 & 6.13 & 6.14 & 12.28 & 6.01 & 5.95 & 11.97 & 6.39 & 6.26 & 12.65 \\
\hline & 5.25 & 12.00 & 17.25 & 9.09 & 4.82 & 13.91 & 8.09 & 5.50 & 13.59 & 4.29 & 6.07 & 10.36 & 8.25 & 5.18 & 13.43 & 8.38 & 9.13 & 17.50 \\
\hline Germ & 6.57 & 4.43 & 11.00 & 4.47 & 6.21 & 10.68 & 9.00 & 6.22 & 15.22 & 5.09 & 8.82 & 13.91 & 6.45 & 7.70 & 14.15 & 7.64 & 5.58 & 13.22 \\
\hline Italy & 4.15 & 7.25 & 11.40 & 6.14 & 4.64 & 10.79 & 7.00 & 5.58 & 12.58 & 4.50 & 10.89 & 15.39 & 6.40 & 3.60 & 10.00 & 4.50 & 7.69 & 12.19 \\
\hline Unite & 5.21 & .15 & 11.37 & 8.55 & 5.90 & 14.45 & 8.70 & 5.67 & 14.37 & 8.10 & 6.09 & 14.19 & 6.27 & 8.60 & 14.87 & 6.33 & 6.82 & 13.15 \\
\hline$\Omega$ th & 5.83 & 4.58 & & 7.74 & 5.60 & 13.34 & 7.49 & 7.50 & 14.99 & 6.27 & 5.76 & 12.03 & 5.84 & 5.89 & 11.72 & 6.32 & 6.04 & 12.36 \\
\hline Asia & 5.24 & 5.72 & 10.96 & 5.99 & 6.64 & 12.63 & 6.08 & 6.53 & 12.60 & 6.75 & 6.76 & 13.51 & 6.63 & 5.44 & 12.07 & 5.56 & 5.73 & 11.29 \\
\hline Kong, & 3.60 & 3.45 & 7.05 & 6.50 & 5.57 & 12.07 & 6.33 & 5.71 & 12.05 & 7.40 & 6.67 & 14.07 & 4.75 & 4.00 & 8.75 & 4.75 & 5.00 & 9.75 \\
\hline Israel & 6.67 & 3.64 & 10.31 & 5.85 & 5.64 & 11.49 & 4.79 & 5.79 & 10.57 & 5.07 & 5.79 & 10.86 & 6.29 & 4.36 & 10.64 & 5.54 & 5.69 & 11.23 \\
\hline Japan & 6.00 & 10.50 & 16.50 & 6.17 & 8.33 & 14.50 & 7.83 & 6.33 & 14.17 & 4.71 & 6.43 & 11.14 & 8.00 & 5.17 & 13.17 & 4.63 & 5.88 & 10.50 \\
\hline Korea & 4.36 & 6.46 & 10.82 & 5.83 & 7.08 & 12.92 & 6.00 & 5.31 & 11.31 & 6.15 & 6.33 & 12.49 & 8.89 & 8.22 & 17.11 & 6.75 & 6.08 & 12.83 \\
\hline Singapore & 5.56 & 4.56 & 10.11 & 5.63 & 6.57 & 12.20 & 5.43 & 9.50 & 14.93 & 10.40 & 8.60 & 19.00 & 5.25 & 5.44 & 10.69 & 6.13 & 6.00 & 12.13 \\
\hline North America & 7.92 & 8.67 & 16.58 & 6.03 & 6.23 & 12.26 & 6.38 & 7.29 & 13.68 & 4.44 & 5.78 & 10.22 & 8.76 & 8.31 & 17.07 & 7.66 & 9.96 & 17.62 \\
\hline Canada & 7.50 & 8.78 & 16.28 & 5.83 & 7.00 & 12.83 & 6.56 & 9.67 & 16.22 & 4.31 & 5.13 & 9.44 & 9.13 & 9.71 & 18.84 & 8.50 & 13.43 & 21.93 \\
\hline United States & 8.33 & 8.56 & 16.89 & 6.23 & 5.46 & 11.69 & 6.21 & 4.92 & 11.14 & 4.57 & 6.43 & 11.00 & 8.40 & 6.90 & 15.30 & 6.82 & 6.50 & 13.32 \\
\hline Oceania & 6.01 & 4.77 & 10.78 & 5.30 & 5.97 & 11.27 & 5.55 & 5.92 & 11.47 & 6.81 & 5.36 & 12.17 & 5.40 & 6.35 & 11.76 & 3.69 & 7.96 & 11.65 \\
\hline Australia & 7.57 & 5.29 & 12.86 & 5.60 & 5.27 & 10.87 & 4.50 & 5.17 & 9.67 & 8.75 & 5.71 & 14.46 & 6.38 & 7.33 & 13.71 & 4.50 & 8.78 & 13.28 \\
\hline New Zealand & 4.44 & 4.25 & 8.69 & 5.00 & 6.67 & 11.67 & 6.60 & 6.67 & 13.27 & 4.88 & 5.00 & 9.88 & 4.43 & 5.38 & 9.80 & 2.88 & 7.14 & 10.02 \\
\hline
\end{tabular}

Note: Other Europe include Austria, Belgium, the Czech Republic, Denmark, Estonia, Finland, Greece, Iceland, Ireland, Latvia, Lithuania, the Netherlands, Norway, Portugal, Slovakia, Slovenia, Spain, Swede

and Switzerland. Advanced economies is abbreviated to AD Economies. Direct investment, portfolio investment and other investment are abbreviated to DI, PI and OI, respectively. (1) is the expansion phase 
Table 2. Duration of capital flows in emerging and developing economies (unit: quarter).

\begin{tabular}{|c|c|c|c|c|c|c|c|c|c|c|c|c|c|c|c|c|c|c|}
\hline \multirow[b]{2}{*}{ EM Economies } & \multicolumn{3}{|c|}{ Net Flows } & \multicolumn{3}{|c|}{ Gross Inflows } & \multicolumn{3}{|c|}{ Gross Outflows } & \multicolumn{3}{|c|}{ Net DI } & \multicolumn{3}{|c|}{ Net PI } & \multicolumn{3}{|c|}{ Net OI } \\
\hline & $(1)$ & $(2)$ & (3) & $(1)$ & $(2)$ & (3) & $(1)$ & $(2)$ & (3) & $(1)$ & $(2)$ & $(3)$ & $(1)$ & $(2)$ & $(3)$ & $(1)$ & $(2)$ & $(3)$ \\
\hline Total Average & 6.07 & 4.86 & 10.94 & 6.82 & 6.41 & 13.22 & 6.53 & 6.93 & 13.46 & 5.87 & 6.51 & 12.39 & 6.21 & 6.61 & 12.81 & 7.31 & 6.16 & 13.48 \\
\hline Europe & .34 & 4.78 & 0.12 & 6.72 & 6.81 & 3.53 & 5.72 & 7.75 & 14.46 & 5.63 & 7.42 & 13.05 & 7.17 & 6.89 & 14.06 & 8.59 & 3.35 & 11.94 \\
\hline Belarus & 5.25 & 6.25 & 11.50 & 9.17 & 6.17 & 15.33 & 4.88 & 6.43 & 11.30 & 6.00 & 8.50 & 14.50 & 7.71 & 6.17 & 13.88 & 8.00 & 7.00 & 15.00 \\
\hline Bulgaria & 5.20 & 3.60 & 8.80 & 7.00 & 5.43 & 12.43 & 6.17 & 9.50 & 15.67 & 5.00 & 13.00 & 18.00 & 4.63 & 6.88 & 11.50 & 5.57 & 7.29 & 12.86 \\
\hline Russia & 5.20 & 5.33 & 10.53 & 7.11 & 4.38 & 11.49 & 6.50 & 5.22 & 11.72 & 4.75 & 7.86 & 12.61 & 6.00 & 6.29 & 12.29 & 5.00 & 7.14 & 12.14 \\
\hline Other Europe & 5.39 & 4.66 & 10.05 & 6.27 & 7.45 & 13.72 & 7.09 & 8.05 & 15.13 & 5.79 & 6.40 & 12.20 & 7.62 & 7.08 & 14.70 & 9.61 & 1.73 & 11.34 \\
\hline Asia & 6.49 & 4.81 & 11.30 & 6.68 & 6.10 & 12.78 & 6.24 & 6.20 & 12.44 & 6.23 & 5.80 & 12.03 & 5.47 & 6.60 & 12.07 & 5.72 & 6.82 & 12.54 \\
\hline China & 7.60 & 7.17 & 14.77 & 8.00 & 6.00 & 14.00 & 6.00 & 7.67 & 13.67 & 5.00 & 9.83 & 14.83 & 5.38 & 4.13 & 9.50 & 5.20 & 9.33 & 14.53 \\
\hline India & 6.88 & 4.13 & 11.00 & 5.13 & 5.88 & 11.00 & 5.11 & 5.25 & 10.36 & 5.33 & 4.44 & 9.78 & 5.50 & 6.43 & 11.93 & 7.57 & 4.25 & 11.82 \\
\hline Malaysia & 5.22 & 3.38 & 8.60 & 7.50 & 10.00 & 17.50 & 4.00 & 4.22 & 8.22 & 8.40 & 6.60 & 15.00 & 4.50 & 11.0 & 15.50 & 6.33 & 7.40 & 13.73 \\
\hline Thailand & 8.38 & 5.00 & 13.38 & 10.00 & 11.60 & 21.60 & 6.38 & 5.78 & 12.15 & 4.78 & 6.67 & 11.44 & 5.38 & 7.38 & 12.75 & 5.90 & 4.89 & 10.79 \\
\hline Other Asia & 0 & 4.75 & 10.94 & 6.12 & 4.80 & 10.92 & 6.74 & 6.47 & 21 & 6.43 & 5.18 & 11.62 & 5.63 & 6.24 & 11.87 & 5.42 & 7.02 & 12.44 \\
\hline South America & 6.94 & 5.29 & 12.22 & 8.28 & 6.84 & 15.12 & 6.31 & 6.25 & 12.56 & 5.61 & 6.32 & 11.93 & 5.82 & 6.69 & 12.51 & 9.14 & 10.05 & 19.19 \\
\hline Argentina & 7.75 & 5.75 & 13.50 & 11.33 & 8.50 & 19.83 & 8.50 & 7.33 & 15.83 & 3.83 & 5.83 & 9.67 & 6.75 & 7.50 & 14.25 & 5.20 & 5.33 & 10.53 \\
\hline Brazil & 5.08 & 4.23 & 9.31 & 5.00 & 5.20 & 10.20 & 7.00 & 7.00 & 14.00 & 5.33 & 7.00 & 12.33 & 4.25 & 8.13 & 12.38 & 5.27 & 4.33 & 9.61 \\
\hline Chile & 4.78 & 7.22 & 12.00 & 13.67 & 5.80 & 19.47 & 6.88 & 6.50 & 13.38 & 8.38 & 5.86 & 14.23 & 5.63 & 7.63 & 13.25 & 20.00 & 27.50 & 47.50 \\
\hline Other South America & 5.87 & 5.73 & 11.60 & 10.00 & 6.50 & 16.50 & 7.46 & 6.94 & 14.40 & 5.85 & 6.23 & 12.08 & 5.54 & 7.75 & 13.29 & 10.16 & 12.39 & 22.55 \\
\hline North America: Mexico & 4.08 & 4.58 & 8.67 & 4.42 & 4.25 & 8.67 & 6.44 & 5.50 & 11.94 & 4.33 & 7.88 & 12.21 & 6.50 & 5.56 & 12.06 & 5.17 & 9.57 & 14.74 \\
\hline Africa: South Africa & 6.50 & 4.50 & 11.00 & 4.47 & 5.67 & 10.13 & 9.00 & 11.71 & 20.71 & 7.18 & 4.91 & 12.09 & 6.33 & 4.50 & 10.83 & 5.06 & 4.24 & 9.30 \\
\hline
\end{tabular}

Brazil and Chile. Emerging and developing economies is abbreviated to EM Economies. 


\subsubsection{Amplitude}

Next, our focus shifts towards the amplitude of capital flows cycle, the greater the amplitude the more volatile the capital flows. As we can see from Table 3, the amplitude of capital flows cycle is relatively high in some small economies that play the role of international financial centers, such as Hong Kong, China (23.94 percent) and Singapore (25.15 percent), while that of some large advanced countries also equipped with strong financial markets is relatively low, such as the United States (3.98 percent), Canada (6.55 percent), the United Kingdom (7.78 percent) and Italy (7.16 percent). For each of the three types of capital flows, the amplitude of other investment is the highest in most countries, followed by portfolio investment and direct investment. In addition, the results show that the amplitude of the three types of capital flows in Hong Kong, China is very high, which is closely related to its critical role in the international financial system?

For emerging and developing economies (see Table 4), the amplitude of gross capital inflows cycles is generally higher than that of gross capital outflows in most countries, which implies that the absorption ability of capital is stronger than the outward expansion ability in those countries. Some large economies, such as China and India, have lower amplitude of capital inflows cycle than small economies, such as Malaysia. For each of the three types of capital flows, the amplitude of other investment and portfolio investment is generally higher than that of direct investment in most countries. For direct investment, the average amplitude of cycles in the Asian region is the highest, and for other investment and portfolio investment the average amplitude of cycles in the European region is the highest. The results reflect that Asian countries have greater economic development potential, which makes them more advantageous for long-term investments, while European economies have more open financial markets, which makes them more advantageous for short-term capital investments.

\subsubsection{Speed}

The speed is the amplitude per one unit and indicates the volatility of capital flows per unit of time. The faster the speed, the more likely a crisis will occur in countries with immature financial systems, especially in a contraction phase of capital flows.

In advanced economies (see Table 5), we found that the speed of capital flows in some small economies is relatively fast. Among them, the speed of capital flows in Hong Kong, China (6.79 percent) is the fastest, followed by Singapore (5.06 percent), while the large economies, such as the United States, Canada, France and Italy, have a slow speed of capital flows. In addition, the speed of the capital flows (net and gross) cycles in most economies in the contraction period is obviously faster than in the expansion period, which means the "flight to safety"s and "home bias"4 may occur simultaneously during a crisis in advanced countries with safer financial products and lower investment costs. For the three types of capital flows, the speed of other investment and portfolio investment is significantly faster than that of direct investment. In emerging and developing economies (see Table 6), the speed of the capital flows cycle in China and India is relatively slow, while those of Malaysia and Thailand are relatively fast, and it is faster in the contraction period than in the expansion period, implying that these countries are more vulnerable to sudden stops of capital flows. For the three types of capital flows, the speed of other investment and portfolio investment is significantly faster than that of direct investment.

\footnotetext{
${ }^{3}$ See Vayanos (2004); Caballero \& Krishnamurthy (2008) and Baele, Bekaert, Inghelbrecht, \& Wei (2020) for details

${ }^{4}$ See Giannetti \& Laeven (2012) and Schmidt \& Zwick (2015) for details.
} 
Table 3. Amplitude of capital flows in advanced economies (unit: percent).

\begin{tabular}{|c|c|c|c|c|c|c|c|c|c|c|c|c|c|c|c|c|c|c|}
\hline \multirow[b]{2}{*}{ AD Economies } & \multicolumn{3}{|c|}{ Net Flows } & \multicolumn{3}{|c|}{ Gross Inflows } & \multicolumn{3}{|c|}{ Gross Outflows } & \multicolumn{3}{|c|}{ Net DI } & \multicolumn{3}{|c|}{ Net PI } & \multicolumn{3}{|c|}{ Net OI } \\
\hline & ( 1 ) & $(2)$ & $(3)$ & ( 1 ) & $(2)$ & ( 3 ) & ( 1 ) & $(2)$ & ( 3 ) & $(1)$ & $(2)$ & $(3)$ & ( 1 ) & $(2)$ & $(3)$ & ( 1 ) & $(2)$ & $(3)$ \\
\hline Total Avera & 17.98 & 18.47 & 18.22 & 58.22 & 62.37 & 60.29 & 59.35 & 62.89 & 61.12 & 15.94 & 15.70 & 15.82 & 28.19 & 29.17 & 28.68 & 32.27 & 32.18 & 32.23 \\
\hline Europe & 9.14 & 19.49 & 19.32 & 61.60 & 67.05 & 64.32 & 62.56 & 67.23 & 64.89 & 16.25 & 16.20 & 16.23 & 30.12 & 31.27 & 30.70 & 34.18 & 33.84 & 34.01 \\
\hline France & 6.77 & 6.61 & 6.69 & 36.79 & 32.62 & 34.71 & 35.92 & 33.36 & 34.64 & 4.77 & 5.13 & 4.95 & 11.87 & 11.82 & 11.85 & 14.26 & 14.95 & 14.61 \\
\hline Germany & 8.18 & 8.77 & 8.47 & 15.90 & 16.36 & 16.13 & 24.62 & 23.94 & 24.28 & 6.50 & 5.79 & 6.15 & 12.20 & 13.13 & 12.66 & 10.14 & 10.40 & 10.27 \\
\hline Italy & .90 & 7.42 & .16 & 16.36 & 16.87 & 16.62 & 16.15 & 16.42 & 16.28 & 3.04 & 3.23 & 3.13 & 11.99 & 11.44 & 11.72 & 13.05 & 13.21 & 13.13 \\
\hline United Kingdom & 7.99 & 7.57 & 7.78 & 56.69 & 59.97 & 58.33 & 66.44 & 71.24 & 68.84 & 15.08 & 14.17 & 14.62 & 26.69 & 27.81 & 27.25 & 26.07 & 25.79 & 25.93 \\
\hline Other Europe & 21.60 & 22.00 & 21.80 & 67.95 & 74.54 & 71.24 & 68.19 & 73.75 & 70.97 & 18.13 & 18.12 & 18.13 & 33.16 & 34.47 & 33.82 & 38.03 & 37.58 & 37.80 \\
\hline Asia & 15.83 & 17.10 & 16.46 & 66.17 & 66.27 & 66.22 & 67.43 & 67.58 & 67.51 & 19.20 & 17.95 & 18.57 & 26.82 & 27.65 & 27.24 & 33.87 & 35.05 & 34.46 \\
\hline Hong Kong, & 3.28 & 24.60 & 23.94 & 149.86 & 139.82 & 144.84 & 142.60 & 134.40 & 138.50 & 52.34 & 48.48 & 50.41 & 62.87 & 63.98 & 63.43 & 81.95 & 79.62 & 80.78 \\
\hline Israel & 15.58 & 16.33 & 15.95 & 14.91 & 14.76 & 14.83 & 18.92 & 19.56 & 19.24 & 7.65 & 6.93 & 7.29 & 9.82 & 10.19 & 10.00 & 14.15 & 15.91 & 15.03 \\
\hline Japan & 8.46 & 8.64 & 8.55 & 15.31 & 15.87 & 15.59 & 16.17 & 16.22 & 16.19 & 2.40 & 2.81 & 2.61 & 14.09 & 15.15 & 14.62 & 11.84 & 11.26 & 11.55 \\
\hline Korea & .28 & 9.17 & & 10.63 & 11.57 & 11.10 & 4.99 & & 4.70 & 1.26 & 1.43 & 1.35 & 5.47 & 5.62 & 5.55 & 8.46 & 9.73 & 9.10 \\
\hline Singapore & 23.56 & 26.75 & 25.15 & 140.15 & 149.32 & 144.73 & 154.46 & \begin{tabular}{|l|}
163.33 \\
\end{tabular} & 158.89 & 32.33 & 30.08 & 31.20 & 41.85 & 43.34 & 42.59 & 52.96 & 58.73 & 55.84 \\
\hline North America & 5.27 & 5.27 & 5.27 & 10.39 & 10.33 & 10.36 & 11.19 & 11.35 & 11.27 & 3.96 & 3.96 & 3.96 & 7.18 & 7.05 & 7.11 & 6.97 & 6.96 & 6.96 \\
\hline Canada & 6.32 & 6.79 & 6.55 & 12.14 & 12.25 & 12.20 & 13.90 & 13.62 & 13.76 & 5.28 & 5.28 & 5.28 & 9.43 & 9.26 & 9.34 & 9.67 & 9.84 & 9.76 \\
\hline United State & 4.21 & 3.74 & 3.98 & 8.64 & 8.41 & 8.53 & 8.49 & 9.09 & 8.79 & 2.64 & 2.64 & 2.64 & 4.93 & 4.84 & 4.89 & 4.28 & 4.07 & 4.17 \\
\hline Oceania & 13.05 & 13.63 & 13.34 & 16.48 & 17.37 & 16.92 & 19.08 & 19.12 & 19.10 & 8.00 & 8.02 & 8.01 & 15.31 & 15.36 & 15.34 & 14.54 & 14.27 & 14.41 \\
\hline Australia & 7.48 & 7.62 & 7.55 & 12.24 & 12.55 & 12.39 & 11.30 & 12.11 & 11.71 & 7.46 & 7.94 & 7.70 & 10.54 & 11.12 & 10.83 & 8.81 & 8.69 & 8.75 \\
\hline New Zealand & 18.63 & 19.64 & 19.13 & 20.72 & 22.19 & 21.45 & 26.85 & 26.13 & 26.49 & 8.53 & 8.10 & 8.32 & 20.09 & 19.60 & 19.84 & 20.28 & 19.85 & 20.06 \\
\hline
\end{tabular}

Note: The amplitude of the cycle is equal to the average of amplitude of the expansion phase and amplitude of the contraction phase. 
Table 4. Amplitude of capital flows in emerging and developing economies (unit: percent).

\begin{tabular}{|c|c|c|c|c|c|c|c|c|c|c|c|c|c|c|c|c|c|c|}
\hline \multirow[b]{2}{*}{ EM Economies } & \multicolumn{3}{|c|}{ Net Flows } & \multicolumn{3}{|c|}{ Gross Inflows } & \multicolumn{3}{|c|}{ Gross Outflows } & \multicolumn{3}{|c|}{ Net DI } & \multicolumn{3}{|c|}{ Net PI } & \multicolumn{3}{|c|}{ Net OI } \\
\hline & (1) & (2) & (3) & (1) & $(2)$ & (3) & (1) & (2) & (3) & (1) & $(2)$ & (3) & (1) & $(2)$ & (3) & (1) & $(2)$ & (3) \\
\hline Total Average & 17.04 & 17.29 & 17.17 & 18.58 & 18.62 & 18.60 & 14.43 & 15.36 & 14.90 & 6.82 & 8.03 & 7.42 & 10.92 & 11.71 & 11.31 & 19.24 & 18.00 & 18.62 \\
\hline Europe & 20.77 & 21.08 & 20.92 & 23.02 & 25.00 & 24.01 & 19.62 & 22.25 & 20.94 & 7.64 & 8.28 & 7.96 & 12.10 & 12.87 & 12.49 & 26.33 & 22.32 & 24.33 \\
\hline Belarus & 21.40 & 19.43 & 20.42 & 20.55 & 17.86 & 19.20 & 10.86 & 11.13 & 10.99 & 7.98 & 8.39 & 8.18 & 5.11 & 5.27 & 5.19 & 18.36 & 15.83 & 17.09 \\
\hline Bulgaria & 23.58 & 24.10 & 23.84 & 20.06 & 21.53 & 20.79 & 19.28 & 17.91 & 18.59 & 13.63 & 15.92 & 14.77 & 10.95 & 11.07 & 11.01 & 19.20 & 19.05 & 19.12 \\
\hline Russia & 17.15 & 17.98 & 17.57 & 13.56 & 14.93 & 14.25 & 13.29 & 13.19 & 13.24 & 3.60 & 3.88 & 3.74 & 7.51 & 11.11 & 9.31 & 21.21 & 17.84 & 19.53 \\
\hline Other Europe & 20.80 & 21.32 & 21.06 & 25.15 & 27.96 & 26.55 & 21.82 & 25.76 & 23.79 & 7.31 & 7.80 & 7.55 & 13.92 & 14.46 & 14.19 & 29.21 & 24.36 & 26.79 \\
\hline Asia & 17.08 & 17.39 & 17.24 & 18.91 & 19.30 & 19.11 & 12.17 & 12.26 & 12.21 & 7.00 & 9.46 & 8.23 & 10.76 & 10.65 & 10.70 & 17.87 & 17.84 & 17.85 \\
\hline China & 9.32 & 8.23 & 8.77 & 8.32 & 8.35 & 8.34 & 8.05 & 8.03 & 8.04 & 2.05 & 2.42 & 2.23 & 3.07 & 2.78 & 2.92 & 8.38 & 7.11 & 7.75 \\
\hline India & 6.14 & 6.13 & 6.13 & 5.06 & 4.76 & 4.91 & 3.03 & 3.03 & 3.03 & 1.62 & 1.53 & 1.57 & 3.35 & 3.72 & 3.54 & 5.04 & 4.64 & 4.84 \\
\hline Malaysia & 23.99 & 24.40 & 24.20 & 41.50 & 48.07 & 44.79 & 13.66 & 14.96 & 14.31 & 6.58 & 6.80 & 6.69 & 25.57 & 23.59 & 24.58 & 21.24 & 19.81 & 20.53 \\
\hline Thailand & 15.35 & 18.52 & 16.93 & 19.46 & 18.69 & 19.07 & 13.22 & 12.30 & 12.76 & 5.81 & 5.94 & 5.87 & 5.75 & 6.77 & 6.26 & 11.78 & 12.35 & 12.07 \\
\hline Other Asia & 19.02 & 19.15 & 19.08 & 19.10 & 18.92 & 19.01 & 13.71 & 13.78 & 13.75 & 8.70 & 12.48 & 10.59 & 11.51 & 11.47 & 11.49 & 21.44 & 21.76 & 21.60 \\
\hline South America & 13.49 & 13.64 & 13.56 & 12.72 & 8.08 & 10.40 & 10.90 & 10.46 & 10.68 & 5.16 & 5.20 & 5.18 & 9.13 & 12.37 & 10.75 & 12.47 & 13.45 & 12.96 \\
\hline Argentina & 12.12 & 11.55 & 11.83 & 16.88 & 9.31 & 13.10 & 9.04 & 5.98 & 7.51 & 3.13 & 3.25 & 3.19 & 8.95 & 8.43 & 8.69 & 8.07 & 9.79 & 8.93 \\
\hline Brazil & 8.76 & 8.36 & 8.56 & 8.74 & 8.92 & 8.83 & 4.43 & 4.66 & 4.55 & 3.41 & 3.19 & 3.30 & 5.36 & 9.24 & 7.30 & 9.24 & 8.80 & 9.02 \\
\hline Chile & 16.56 & 17.06 & 16.81 & 13.67 & 5.80 & 3.93 & 21.47 & 21.20 & 21.33 & 10.20 & 11.25 & 10.72 & 12.11 & 12.88 & 12.49 & 21.68 & 24.77 & 23.22 \\
\hline Other South America & 14.99 & 15.61 & 15.30 & 12.17 & 13.97 & 13.07 & 9.78 & 10.23 & 10.01 & 4.54 & 4.15 & 4.34 & 9.61 & 15.64 & 12.62 & 11.69 & 11.94 & 11.82 \\
\hline North America: Mexico & 6.07 & 6.63 & 6.35 & 7.87 & 8.25 & 8.06 & 7.37 & 7.98 & 7.67 & 3.70 & 3.77 & 3.73 & 9.39 & 9.26 & 9.33 & 7.80 & 8.77 & 8.28 \\
\hline Africa: South Africa & 8.02 & 7.20 & 7.61 & 10.57 & 10.41 & 10.49 & 12.10 & 12.55 & 12.32 & 8.09 & 8.17 & 8.13 & 11.31 & 10.82 & 11.06 & 8.65 & 8.41 & 8.53 \\
\hline
\end{tabular}


Table 5. Speed of capital flows in advanced economies (unit: percent).

\begin{tabular}{|c|c|c|c|c|c|c|c|c|c|c|c|c|c|c|c|c|c|c|}
\hline \multirow[b]{2}{*}{ AD Economies } & \multicolumn{3}{|c|}{ Net Flows } & \multicolumn{3}{|c|}{ Gross Inflows } & \multicolumn{3}{|c|}{ Gross Outflows } & \multicolumn{3}{|c|}{ Net DI } & \multicolumn{3}{|c|}{ Net PI } & \multicolumn{3}{|c|}{ Net OI } \\
\hline & ( 1 ) & (2) & ( 3 ) & ( 1 ) & $(2)$ & ( 3 ) & ( 1 ) & $(2)$ & $(3)$ & ( 1 ) & ( 2 ) & ( 3 ) & ( 1 ) & (2) & ( 3 ) & $(1)$ & ( 2 ) & ( 3 ) \\
\hline$\overline{\rho e}$ & 3.23 & 17 & 3.70 & 7.70 & 10.24 & 8.97 & 8.07 & 9.21 & 8.64 & 2.69 & 2.60 & 2.65 & 5.16 & 5.28 & 5.22 & 6.08 & 5.29 & 5.68 \\
\hline & 3.36 & 4.44 & 3.90 & 7.58 & 11.03 & 9.30 & 7.93 & 9.91 & 8.92 & 2.89 & 2.76 & 2.83 & 5.51 & 5.51 & 5.51 & 6.31 & 5.51 & 5.91 \\
\hline $\mathrm{F}$ & .29 & .55 & .92 & 4.05 & 6.77 & 5.41 & 4.44 & 6.07 & 5.25 & 1.11 & 0.85 & 0.98 & 1.44 & 2.28 & 1.86 & 1.70 & 1.64 & 1.67 \\
\hline & 24 & 1.98 & .61 & 3.56 & 2.63 & 3.10 & 2.74 & 3.85 & 3.29 & 1.28 & .66 & 0.97 & 1.89 & 1.71 & 1.80 & 1.33 & 1.86 & 1.60 \\
\hline & 1.00 & - & 1.34 & 2.66 & 3.63 & 0.10 & 2.31 & 2.94 & 2.02 & 0.68 & 0.30 & 0.49 & 1.87 & 0.10 & 2.53 & 2.90 & 1.12 & 2.31 \\
\hline & 1.53 & & 1.38 & 6.63 & 10.16 & 8.40 & 7.64 & 12.57 & 10.10 & 1.86 & 33 & 2.09 & 4.25 & 3.23 & 3.74 & 4.12 & 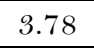 & 3.95 \\
\hline & 3.76 & & 4.44 & 8.28 & 12.13 & 10.20 & 8.70 & 66 & 9.68 & 3.24 & .12 & 3.18 & 6.17 & 6.12 & 6.15 & 7.11 & & 6.65 \\
\hline Asia & 3.27 & 3.94 & 3.61 & 10.96 & 10.79 & 10.88 & 11.56 & 9.50 & 10.53 & 2.48 & 2.53 & 2.50 & 5.03 & 5.98 & 5.51 & 6.45 & 6.40 & 6.43 \\
\hline $\mathrm{n}$ & 6.47 & 7.12 & 6.79 & 23.05 & 25.10 & 24.08 & 22.52 & 23.52 & 23.02 & 7.07 & 7.27 & 7.17 & 13.24 & 16.00 & 14.62 & 17.25 & 15.92 & 16.59 \\
\hline & 2.34 & 4.48 & 3.41 & 2.55 & 2.62 & 2.58 & 3.95 & 3.38 & 3.67 & 1.51 & 1.20 & 1.35 & 1.56 & 2.34 & 1.95 & 2.55 & 2.79 & 2.67 \\
\hline & 1.41 & & 1.12 & 2.48 & 1.90 & 19 & 2.06 & 2.56 & 31 & 1 & 4 & 0.41 & 1.76 & 93 & 2.35 & 2.56 & 1 & 2.24 \\
\hline & 1.90 & & 1.66 & 1.82 & 1.63 & .73 & 0.83 & 0.83 & 0.83 & 0.21 & 0.23 & 0.22 & 0.62 & 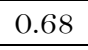 & 0.65 & 1.25 & 1.60 & 1.43 \\
\hline 0 & 4.24 & $5.8^{\circ}$ & 5.06 & 24.92 & 22.72 & 23.82 & 28.45 & 17.19 & 22.82 & 91 & 3.50 & 3.30 & 7.97 & 7.96 & 7.97 & 65 & 9 & 9.22 \\
\hline North & 0.67 & $t=0$ & 0.64 & 1.73 & 1.65 & 1.69 & 1.74 & 1.63 & 1.69 & 0.90 & 0.72 & 0.81 & 0.81 & 0.83 & 0.82 & 0.88 & 0.68 & 0.78 \\
\hline Canada & 0.84 & 0.77 & 0.81 & 2.08 & 1.75 & 1.92 & 2.12 & 1.41 & 1.76 & 1.22 & 1.03 & 1.13 & 1.03 & 0.95 & 0.99 & 1.14 & 0.73 & 0.94 \\
\hline United St & 0.51 & 0.44 & 0.47 & 1.39 & 1.54 & 1.46 & 1.37 & 1.85 & 1.61 & 0.58 & 0.41 & 0.49 & 0.59 & 0.70 & 0.64 & 0.63 & 0.63 & 0.63 \\
\hline & 2.59 & 3.03 & 2.81 & 3.16 & 2.85 & 3.01 & 3.29 & 3.13 & 3.21 & 1.30 & 1.51 & 1.40 & 3.09 & 2.58 & 2.84 & 4.51 & 1.88 & 3.19 \\
\hline Australia & 0.99 & 1.44 & 1.22 & 2.19 & 2.38 & 2.28 & 2.51 & 2.34 & 2.43 & 0.85 & 1.39 & 1.12 & 1.65 & 1.52 & 1.58 & 1.96 & 0.99 & 1.47 \\
\hline New Zealand & 4.19 & 4.62 & 4.41 & 4.14 & 3.33 & 3.74 & 4.07 & 3.92 & 3.99 & 1.75 & 1.62 & 1.69 & 4.54 & 3.65 & 4.09 & 7.05 & 2.78 & 4.92 \\
\hline
\end{tabular}


Table 6. Speed of capital flows in emerging and developing economies (unit: percent).

\begin{tabular}{|c|c|c|c|c|c|c|c|c|c|c|c|c|c|c|c|c|c|c|}
\hline \multirow[b]{2}{*}{ EM Economies } & \multicolumn{3}{|c|}{ Net Flows } & \multicolumn{3}{|c|}{ Gross Inflows } & \multicolumn{3}{|c|}{ Gross Outflows } & \multicolumn{3}{|c|}{ Net DI } & \multicolumn{3}{|c|}{ Net PI } & \multicolumn{3}{|c|}{ Net OI } \\
\hline & (1) & (2) & (3) & $(1)$ & $(2)$ & (3) & $(1)$ & (2) & (3) & $(1)$ & (2) & $(3)$ & $(1)$ & $(2)$ & (3) & $(1)$ & $(2)$ & (3) \\
\hline Total Average & 3.11 & 3.74 & 3.42 & 3.08 & 3.15 & 3.11 & 2.42 & 2.32 & 2.37 & 1.20 & 1.38 & 1.29 & 1.81 & 1.80 & 1.80 & 2.71 & 2.46 & 2.58 \\
\hline Europe & 10 & 4.54 & 4.32 & 3.90 & 4.16 & 4.03 & 3.14 & 3.07 & 3.10 & 1.42 & 1.23 & .33 & 1.77 & .84 & 1.80 & .08 & .10 & 3.09 \\
\hline & .00 & .1 & 3.59 & 2.24 & $\mathrm{O}$ & 2.5 & 2. & 1.73 & 1.98 & 1.33 & 0.99 & 1.16 & 0.66 & 0.85 & 0.76 & 2.29 & .26 & 2.28 \\
\hline & . & .00 & 0.01 & 2.87 & 3.97 & 3.42 & 0.10 & 1.89 & 2.51 & 2.73 & 1.22 & 1.97 & 2.37 & 1.61 & 1.99 & 3.45 & 2.61 & 3.03 \\
\hline & 3.30 & 3.37 & 3.33 & 1.91 & 3.41 & 2.66 & 2 & 2.53 & 2.28 & 0.76 & 0.49 & 0.63 & 1.25 & 1.77 & 1.51 & 4.24 & .50 & 3.37 \\
\hline Other Eur & 4.15 & 4.60 & 4.38 & 4.57 & 4.47 & 4.52 & 3.43 & 3.50 & 3.47 & 1.34 & 1.37 & 1.36 & 1.91 & 2.02 & 1.97 & 2.97 & 3.38 & 3.17 \\
\hline Asia & 3.03 & 3.95 & 3.49 & 3.09 & 3.36 & 3.23 & 2.27 & 2.10 & 2.18 & 1.18 & 1.82 & 1.50 & 1.99 & 1.63 & 1.81 & 3.14 & 2.41 & 2.78 \\
\hline China & .23 & 1.15 & 1.19 & 1.04 & 1.39 & 1.22 & 1.34 & 1.05 & 1.19 & 0.41 & 0.25 & 0.33 & 0.57 & 0.67 & 0.62 & 1.61 & 0.76 & 1.19 \\
\hline & & & & & & & & & & 30 & & & 1 & 58 & & & 09 & 0.88 \\
\hline Mala & 4.59 & 7.23 & 5.91 & 5.5 & 4.81 & 5.1 & 3.4 & 3.54 & 3.48 & 0.78 & 1.03 & 0.91 & 5.68 & 2.14 & 3.91 & 3.35 & 2.68 & 3.02 \\
\hline Thailand & 1.83 & 3.70 & 2.77 & 1.95 & 1.61 & 1.78 & 2.07 & 2.13 & 2.10 & 1.22 & 0.89 & 1.05 & 1.07 & 0.92 & 0.99 & 2.00 & 2.53 & 2.26 \\
\hline Other Asia & 3.55 & 4.26 & 3.90 & 3.49 & 4.05 & 3.77 & 2.51 & 2.25 & 2.38 & 1.46 & 2.50 & 1.98 & 1.99 & 1.94 & 1.97 & 3.85 & 2.77 & 3.31 \\
\hline South America & 1.98 & 2.58 & 2.28 & 1.82 & 1.18 & 1.50 & 1.79 & 1.73 & 1.76 & 0.89 & 0.85 & 0.87 & 1.56 & 2.00 & 1.78 & .48 & 1.68 & 1.58 \\
\hline Argentina & 1.56 & 2.01 & 1.79 & 1.4 & 1.10 & 1.2 & 1.06 & 0.82 & 0.94 & 0.82 & 0.56 & 0.69 & 1.33 & 1.12 & 1.23 & 1.55 & 1.84 & 1.69 \\
\hline Brazil & .72 & 1.97 & 1.85 & 1.7 & 1.72 & 1.7 & 0.6 & 0.67 & 0.65 & 0.64 & 0.46 & 0.55 & 1.26 & 1.14 & 1.20 & 1.75 & 2.03 & 1.89 \\
\hline Chile & 3.47 & 2.36 & 2.91 & 1.00 & 1.00 & 0.00 & 3.12 & 3.26 & 3.19 & 1.22 & 1.92 & 1.57 & 2.15 & 1.69 & 1.92 & 1.08 & 0.90 & 0.99 \\
\hline Other South America & 1.58 & 3.29 & 2.43 & 2.43 & 2.03 & 2.23 & 2.07 & 1.95 & 2.01 & 0.90 & 0.66 & 0.78 & .54 & .02 & 2.28 & .50 & .81 & 1.66 \\
\hline North America: Mexico & 1.49 & 1.45 & 1.47 & 1.78 & 1.94 & 1.86 & 1.14 & 1.45 & 1.30 & 0.85 & 0.48 & 0.67 & 1.45 & 1.67 & 1.56 & 1.51 & 0.92 & 1.21 \\
\hline Africa: South Africa & 1.23 & 1.60 & 1.42 & 2.37 & 1.84 & 2.10 & 1.34 & 1.07 & 1.21 & 1.13 & 1.66 & 1.40 & 1.79 & 2.40 & 2.09 & 1.71 & 1.99 & 1.85 \\
\hline
\end{tabular}




\subsection{Cyclical Characteristics of Capital Flows in Different Regions}

Considering that capital flows might have spatial clustering characteristics, we further explored the cyclical features of capital flows in different regions (see Figures 2, 3 and 4). Specifically, we first calculated regional capital flows weighted by GDP, then removed the trend term of the regional capital flows using the band pass (BP) filtering method, finally identifying the turning points (troughs and peaks) of the cycles by adopting the BBQ algorithm.

For advanced economies (see Figure 2, left panel), the expansion period of the net capital flows cycle lasted 20 quarters (Q3 1982 to Q3 1987), followed by a contraction period lasting nine quarters (Q4 1987 to Q4 1989). Then it entered the next expansion period lasting 16 quarters from $Q^{4} 1989$ to $Q^{4} 1993$, which might be the result of deregulation and monetary tightening in the United States and developed European countries in the 1980s and early 1990s. Following this, advanced economies experienced two long periods of contraction of net capital flows cycles from the mid-1990s to the mid-2000s (Q4 1993 to Q3 1998 and Q4 2000 to Q4 2004), the former mainly due to loose monetary policy in the United States and the European currency crisis, and the latter can be explained by the rapid development of emerging economies in the early 2000s coupled with their reform and openness policies, which prompted a large amount of international capital to shift to emerging economies. Afterwards, the advanced economies experienced an extended period of contraction of capital flows cycles in the 2010s (Q2 2011 to Q3 2016), which was mainly driven by the 2010 European debt crisis.

More specifically, by region (see Figure 3), the volatility of net capital flows in Asia has increased significantly since 1995, and the durations of expansion and contraction periods have shortened; in Europe, the net capital flows cycles have been more volatile since Q3 2006, and the duration of both expansionary and contractionary periods has been significantly shorter; North America experienced a period of expansion and contraction of net capital flows cycle with high amplitude around the 2008 global financial crisis, followed by a return to pre-crisis levels of volatility of capital flows; in Oceania, the durations of net capital flows cycles also display a shorting trend after a long contraction period (Q4 2005 to $\left.\mathrm{Q}^{4} 2010\right)$.

For emerging and developing economies (see Figure 2, right panel), there has been a long period of expansion (Q1 1989 to Q2 1996) and a long period of contraction (Q2 1996 to Q4 2005) since the 1980s, the former was due to the rapid economic growth and economic liberalization of the major emerging and developing countries in the early 1990s, especially China and India, and the latter was probably driven by tight monetary policy in developed economies. The duration of net capital flows cycle has been significantly shorter since mid-200os.

By region (see Figure 4), Asia follows a pattern similar to emerging and developing economies as a whole; in Europe, the volatility of net capital flows has increased significantly compared with the pre-2008 global financial crisis; in South America, the duration of the capital flows cycle has tended to shorten in recent years; in North America, net capital flows have become more volatile since 2008, and the duration of the expansion and contraction periods has shortened; in Africa, the duration of net capital flows cycle also tends to shorten, but the amplitude does not change much.

In summary, a common feature is that the duration of the net capital flows cycle significantly shortened in most regions after the 2008 global financial crisis. The difference is that the amplitude of the net capital flows cycle has enlarged significantly for emerging and developing economies in contrast to fewer changes for developed economies. 
Advanced economies

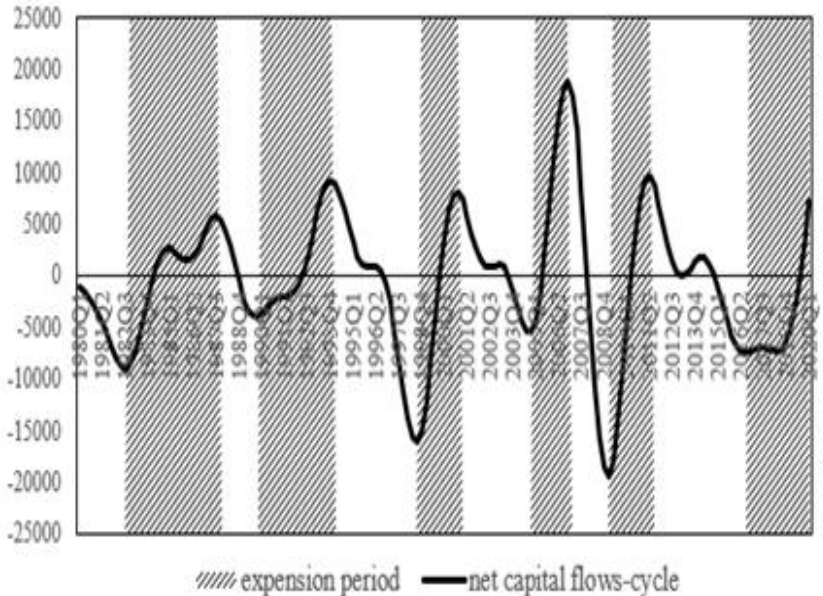

Emerging and developing economies

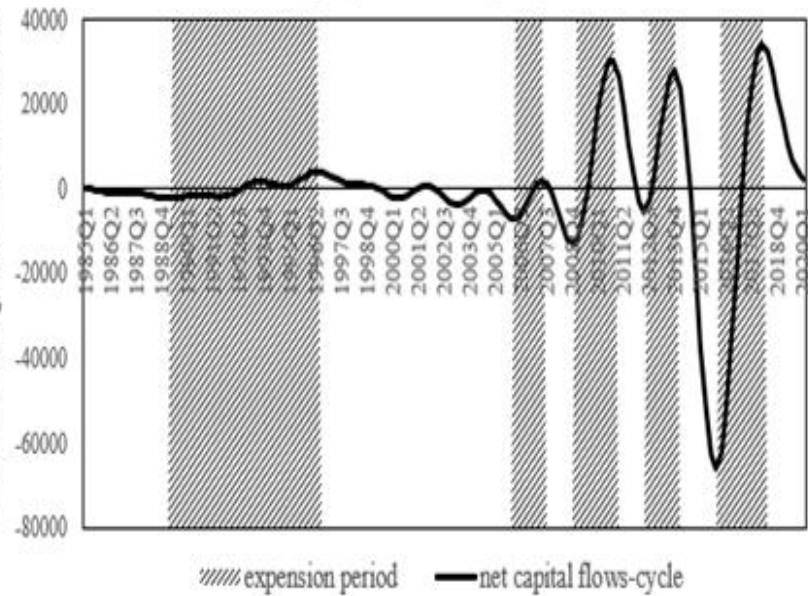

Figure 2. Cyclical characteristics of capital flows in advanced economies and emerging and developing economies.

Source: Sample data start and end times for different economies: Advanced economies (Q1 1980 to Q1 2020), emerging and developing economies (Q1 1985 to Q1 2020)

Calculated by the authors in MATLAB and EXCEL according to the selected capital flows data from the IMF Balance of Payments Statistics

Asia

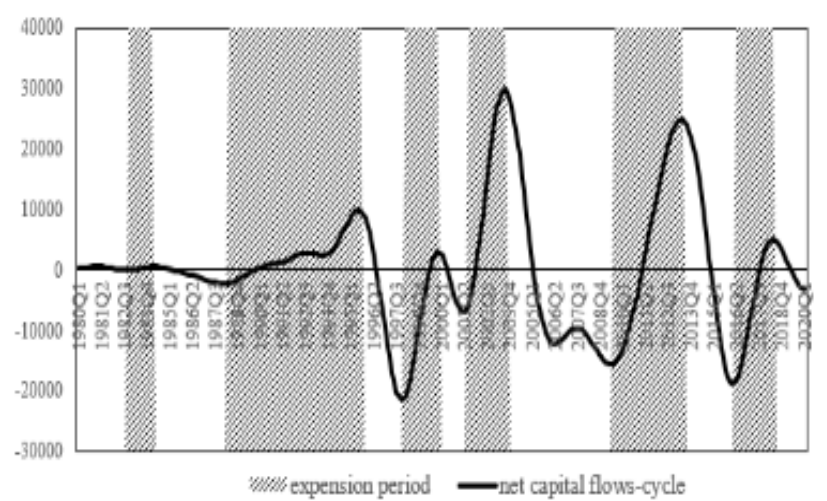

North America

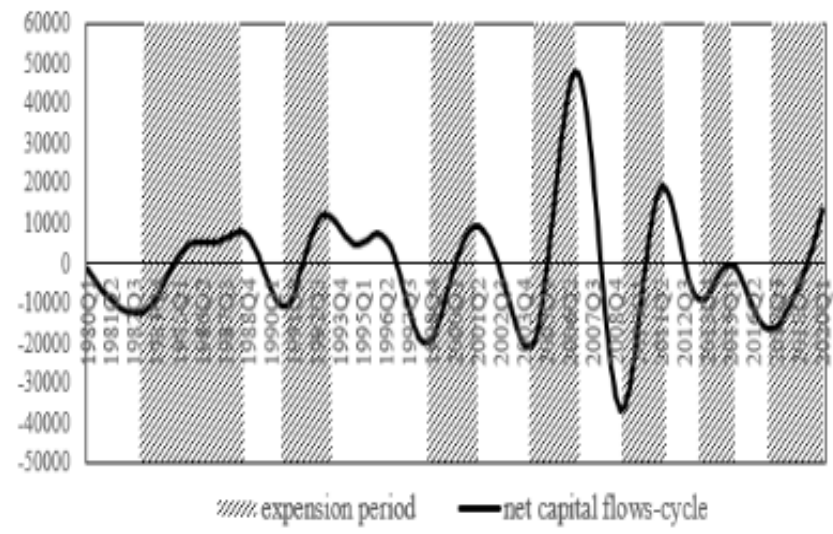

Europe

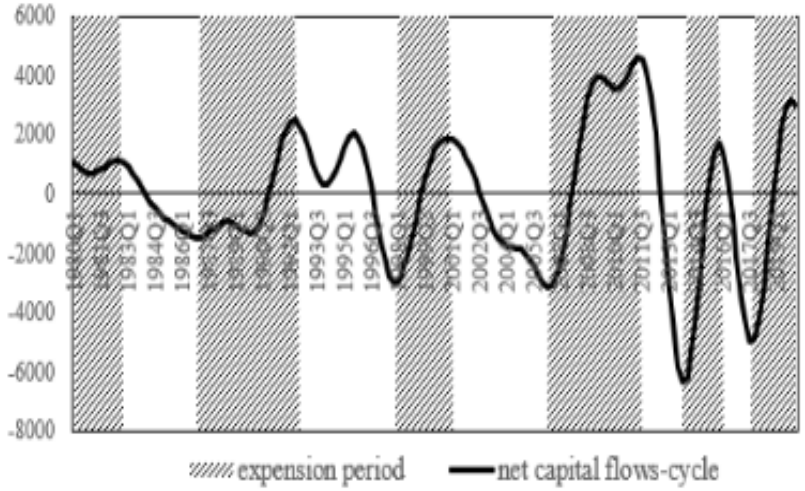

Oceania

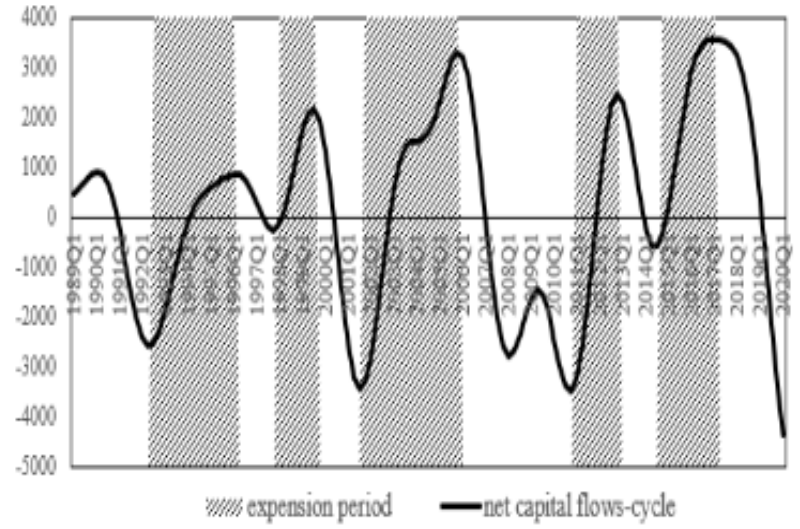

Figure 3. Cyclical characteristics of capital flows in different regions of advanced economies.

Note: Sample data start and end times for different regions of advanced economies: Asia (Q1 1980 to Q1 2020), Europe (Q1 1980 to Q1 2020$)$, North America (Q1 1980 to Q1 2020), Oceania (Q1 1989 to Q1 2020). 

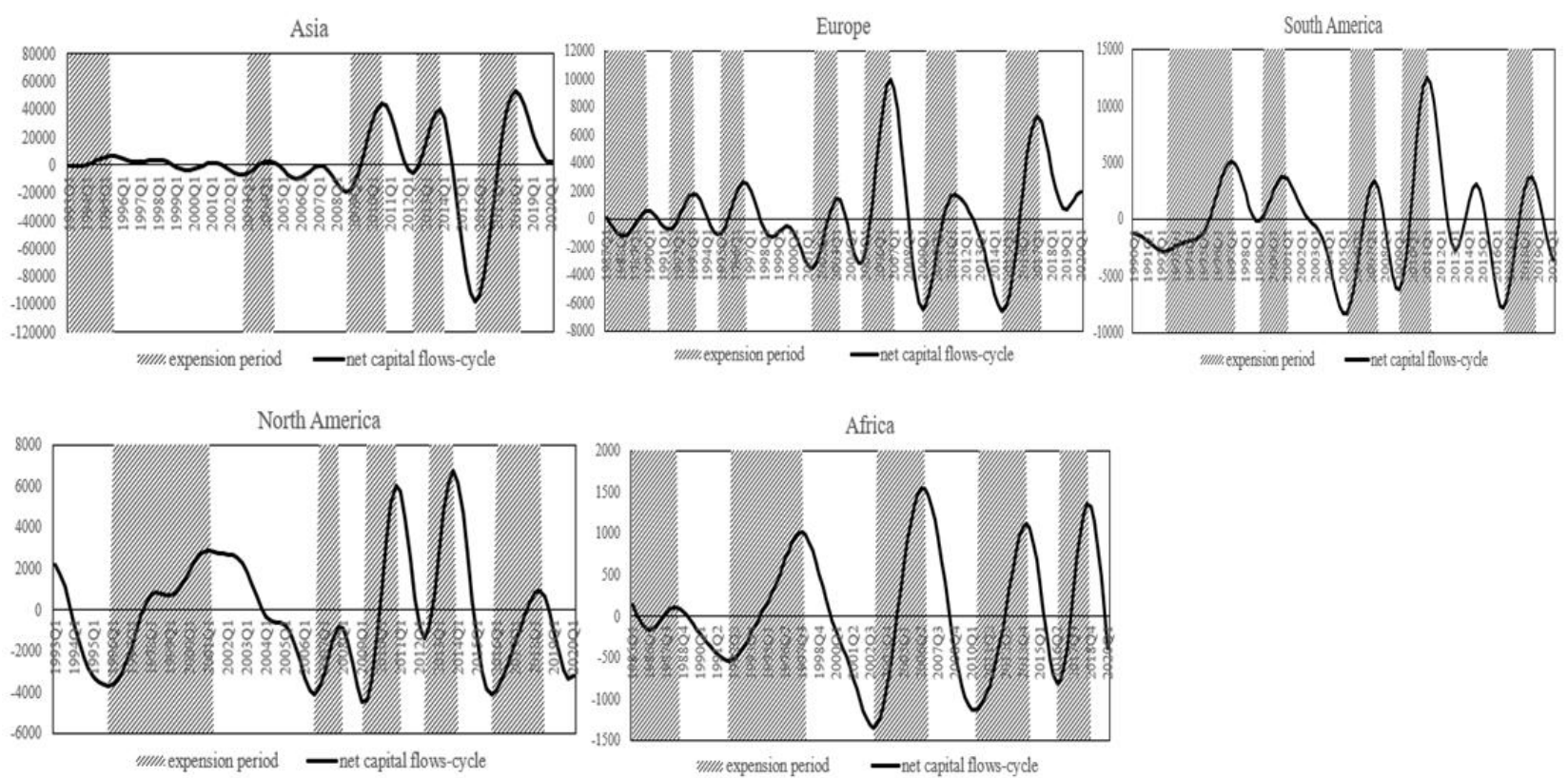

Figure 4. Cyclical characteristics of capital flows in different regions of emerging and developing economies.

Note: Sample data start and end times for different regions of emerging and developing economies: Asia (Q1 1993 to Q1 2020), Europe (Q1 1987 to Q1 2020), South America (Q1 1990 to Q1 2020), North America (Q1 1993 to Q1 2020), Africa (Q1 1985 to Q1 2020).

\section{THE REGIONAL SYNCHRONIZATION OF CAPITAL FLOWS CYCLES}

The study by Rey (2015) points out the basic fact that capital flows in different regions are synergistic. By calculating the correlation of capital flows across regions or different capital types, she found that the gross capital flows in different regions are generally characterized by co-movement. After the 2008 global financial crisis, emerging markets have become increasingly attractive to international investors, and these countries also increasingly tend to invest abroad (see Obstfeld (2012)). This has led to a greater integration of emerging markets with global markets, characterized by more synergistic capital flows cycles across regions.

To understand the degree of globalization of capital flows cycles, we quantified synchronization of capital flows cycles across regions. Harding \& Pagan (2006) have addressed the synchronization of two cycles, which helps us to understand the degree of co-movement of capital flows (net and gross) across regions. Following Harding \& Pagan (2006), we constructed a synchronization index to examine the synergies between capital flows cycle series, specifically the synchronization index between variables $\mathrm{X}$ and $\mathrm{Y}$ within $\mathrm{t}=1,2,3$, etc. T is denoted by $\rho_{X Y}$, which is calculated as follows:

$$
\rho_{X Y}=\frac{1}{T} \sum_{t=1}^{T}\left[\rho_{t}^{X} \times \rho_{t}^{Y}+\left(1-\rho_{t}^{X}\right) \times\left(1-\rho_{t}^{Y}\right)\right]
$$

In Equation 1, if the value of $\rho_{t}^{X}$ is 0 , this means that cyclical series $\mathrm{X}$ is in a contraction phase; if its value is 1,

it means that the cyclical series $\mathrm{X}$ is in an expansion phase; if $\rho_{t}^{Y}$ equals $\mathrm{O}$, this suggests that cyclical series $\mathrm{Y}$ is in a contraction phase, and if it equals 1, the cyclical series $\mathrm{Y}$ is in an expansion phase. Therefore, the value calculated by Equation 1 is the proportion of the total sample time that the two cyclical series are in the same phase (expansion phase or contraction phase). If the index is closer to 1 , the degree of synchronization of the two cyclical series is higher; on the contrary, if the index tends toward 0 , the degree of synchronization of the two cyclical series is lower. 
According to Equation 1, we first calculate the pairwise synchronization index for 12 capital flows (net and gross) cycle series in four regions (Asia, Europe, North America and Oceania) of advanced economies, and then calculate the pairwise synchronization index for 15 capital flows cycle series in five regions (Asia, Europe, South America, North America and Africa) of emerging and developing economies (see Table 7 and Table 8). In addition, we also calculate the synchronization of capital flows cycles between developed economies and emerging markets across regions (see Table 9).

For advanced economies (see Table 7) in terms of gross capital flows, the interregional synchronization indices all exceed 0.5. In terms of net capital flows, there is a high degree of synchronization between Asia and Oceania and between Europe and North America, with results of 0.71 and 0.72 , respectively, implying that they are at the same phase, 71 percent and 72 percent of the time, respectively. This phenomenon shows a high degree of synchronization of capital flows cycles across regions within advanced economies, which is closely related to high financial integration levels of advanced economies.

For emerging and developing economies (see Table 8) in terms of gross capital flows, the interregional synchronization indices are obviously lower than advanced economies, implying a lower degree of regional financial integration in emerging and developing economies than in developed countries. In terms of net capital flows, the synchronization index of the cycles between Asia and other regions (Europe, South America, North America and Africa) is $0.53,0.61,0.58$ and 0.66, respectively; all exceed 0.5, which means that emerging Asia has strong economic and financial ties with other emerging regions.

Table 7. The synchronization of capital flows cycles across regions within advanced economies.

\begin{tabular}{|c|c|c|c|c|c|c|c|c|c|c|c|c|}
\hline Variable & $(1)$ & $(2)$ & $(3)$ & $(4)$ & $(5)$ & $(6)$ & $(7)$ & $(8)$ & $(9)$ & $(10)$ & $(11)$ & $(12)$ \\
\hline (1) Asia-NCF & 1.00 & & & & & & & & & & & \\
\hline (2) Asia-CIF & 0.68 & 1.00 & & & & & & & & & & \\
\hline (3) Asia-COF & 0.50 & 0.76 & 1.00 & & & & & & & & & \\
\hline (4) Europe-NCF & 0.40 & 0.36 & 0.36 & 1.00 & & & & & & & & \\
\hline (5) Europe-CIF & 0.50 & 0.60 & 0.74 & 0.45 & 1.00 & & & & & & & \\
\hline (6) Europe-COF & 0.53 & 0.71 & 0.87 & 0.32 & 0.87 & 1.00 & & & & & & \\
\hline (7) North America-NCF & 0.41 & 0.42 & 0.55 & 0.72 & 0.66 & 0.53 & 1.00 & & & & & \\
\hline (8) North America-CIF & 0.56 & 0.57 & 0.73 & 0.44 & 0.75 & 0.81 & 0.61 & 1.00 & & & & \\
\hline (9) North America-COF & 0.67 & 0.77 & 0.75 & 0.28 & 0.79 & 0.84 & 0.49 & 0.80 & 1.00 & & & \\
\hline (10) Oceania-NCF & 0.71 & 0.55 & 0.51 & 0.29 & 0.50 & 0.57 & 0.34 & 0.58 & 0.69 & 1.00 & & \\
\hline (11) Oceania-CIF & 0.61 & 0.65 & 0.78 & 0.39 & 0.69 & 0.80 & 0.57 & 0.79 & 0.70 & 0.48 & 1.00 & \\
\hline (12) Oceania-COF & 0.49 & 0.53 & 0.62 & 0.55 & 0.64 & 0.66 & 0.69 & 0.65 & 0.54 & 0.32 & 0.84 & 1.00 \\
\hline
\end{tabular}

In Table 9 we can see that the degree of synchronization of capital flows (net and gross) cycles is very high between advanced Asian economies and any region with emerging and developing economies. This phenomenon is strongly related to the fact that the advanced Asian economies (Hong Kong, China, Japan, Korea, Singapore and Israel) and international financial markets have matured. In addition, emerging and developing South America has a high degree of synchronization of capital flows cycles with advanced Europe and advanced North America. The result may be attributed to most countries in these regions being upper-middle income countries and have relatively open financial markets. Similarly, capital flows cycles between advanced Oceania and emerging and developing Europe are also highly synchronized. 
Table 8. The synchronization of capital flows cycles across regions within emerging and developing economies.

\begin{tabular}{|c|c|c|c|c|c|c|c|c|c|c|c|c|c|c|c|}
\hline Variable & $(1)^{\prime}$ & $(2)^{\prime}$ & $(3)^{\prime}$ & $(4)^{\prime}$ & $(5)^{\prime}$ & $(6)^{\prime}$ & $(7)^{\prime}$ & $(8)^{\prime}$ & $(9)^{\prime}$ & $(10)^{\prime}$ & $(11)^{\prime}$ & $(12)^{\prime}$ & $(13)^{\prime}$ & $(14)^{\prime}$ & $(15)^{\prime}$ \\
\hline (1)'Asia-NCF & 1.00 & & & & & & & & & & & & & & \\
\hline$(\boldsymbol{2})^{\prime}$ Asia-CIF & 0.75 & 1.00 & & & & & & & & & & & & & \\
\hline$(3)^{\prime}$ Asia-COF & 0.51 & 0.71 & 1.00 & & & & & & & & & & & & \\
\hline $\begin{array}{l}(4)^{\prime} \text { Europe- } \\
\text { NCF }\end{array}$ & 0.53 & 0.47 & 0.50 & 1.00 & & & & & & & & & & & \\
\hline $\begin{array}{l}(5)^{\prime} \text { Europe- } \\
\text { CIF }\end{array}$ & 0.59 & 0.54 & 0.54 & 0.74 & 1.00 & & & & & & & & & & \\
\hline 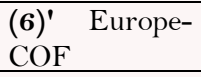 & 0.59 & 0.54 & 0.43 & 0.47 & 0.72 & 1.00 & & & & & & & & & \\
\hline $\begin{array}{lr}(7)^{\prime} \quad \text { South } \\
\text { America-NCF }\end{array}$ & 0.61 & 0.67 & 0.50 & 0.61 & 0.67 & 0.61 & 1.00 & & & & & & & & \\
\hline $\begin{array}{lr}(8)^{\prime} \quad \text { South } \\
\text { America -CIF }\end{array}$ & 0.63 & 0.70 & 0.53 & 0.64 & 0.68 & 0.59 & 0.94 & 1.00 & & & & & & & \\
\hline $\begin{array}{lr}(\mathbf{9})^{\prime} \quad \text { South } \\
\text { America-COF }\end{array}$ & 0.68 & 0.72 & 0.60 & 0.54 & 0.61 & 0.50 & 0.65 & 0.72 & 1.00 & & & & & & \\
\hline $\begin{array}{l}(\mathbf{1 0})^{\prime} \quad \text { North } \\
\text { America-NCF }\end{array}$ & 0.58 & 0.72 & 0.51 & 0.46 & 0.40 & 0.46 & 0.64 & 0.63 & 0.64 & 1.00 & & & & & \\
\hline $\begin{array}{l}(11)^{\prime} \quad \text { North } \\
\text { America-CIF }\end{array}$ & 0.53 & 0.58 & 0.50 & 0.50 & 0.43 & 0.47 & 0.49 & 0.48 & 0.54 & 0.79 & 1.00 & & & & \\
\hline $\begin{array}{l}(\mathbf{1 2})^{\prime} \quad \text { North } \\
\text { America-COF }\end{array}$ & 0.50 & 0.47 & 0.54 & 0.52 & 0.54 & 0.54 & 0.47 & 0.46 & 0.52 & 0.39 & 0.58 & 1.00 & & & \\
\hline $\begin{array}{l}(13)^{\prime} \text { Africa- } \\
\text { NCF }\end{array}$ & 0.66 & 0.58 & 0.56 & 0.50 & 0.71 & 0.76 & 0.63 & 0.64 & 0.63 & 0.44 & 0.52 & 0.49 & 1.00 & & \\
\hline $\begin{array}{l}(14)^{\prime} \text { Africa- } \\
\text { CIF }\end{array}$ & 0.76 & 0.66 & 0.55 & 0.55 & 0.62 & 0.64 & 0.62 & 0.67 & 0.61 & 0.49 & 0.55 & 0.42 & 0.84 & 1.00 & \\
\hline $\begin{array}{l}(\mathbf{1 5})^{\prime} \text { Africa- } \\
\text { COF }\end{array}$ & 0.67 & 0.57 & 0.48 & 0.62 & 0.57 & 0.55 & 0.53 & 0.58 & 0.57 & 0.54 & 0.62 & 0.35 & 0.75 & 0.91 & 1.00 \\
\hline
\end{tabular}

Note: Asia: Armenia, China, Georgia, India, Indonesia, Kazakhstan, Malaysia, Mongolia, Philippines, Thailand, Vietnam; Europe: Belarus, Bulgaria, Croatia, Hungary, Moldova, Poland, Romania, Russia, Turkey, Ukraine; North America: Mexico; South America: Argentina, Brazil, Chile, Colombia, Ecuador; Africa: South Africa.

Table 9. The synchronization of capital flows cycles across regions between advanced economies and emerging and developing economies.

\begin{tabular}{|c|c|c|c|c|c|c|c|c|c|c|c|c|}
\hline EM AD & (1) & $(2)$ & (3) & $(4)$ & (5) & (6) & $(7)$ & (8) & (9) & (10) & (11) & $(12)$ \\
\hline (1)' Asia-NCF & 0.71 & 0.57 & 0.61 & 0.37 & 0.64 & 0.66 & 0.45 & 0.69 & 0.69 & 0.56 & 0.72 & 0.58 \\
\hline$(2)^{\prime}$ Asia-CIF & 0.68 & 0.63 & 0.69 & 0.43 & 0.56 & 0.58 & 0.48 & 0.57 & 0.59 & 0.51 & 0.63 & 0.50 \\
\hline$(3)^{\prime}$ Asia-COF & 0.50 & 0.54 & 0.72 & 0.49 & 0.67 & 0.67 & 0.55 & 0.57 & 0.59 & 0.61 & 0.54 & 0.42 \\
\hline$(4)^{\prime}$ Europe-NCF & 0.55 & 0.56 & 0.56 & 0.45 & 0.67 & 0.67 & 0.51 & 0.70 & 0.62 & 0.62 & 0.67 & 0.64 \\
\hline$(5)^{\prime}$ Europe-CIF & 0.66 & 0.67 & 0.60 & 0.28 & 0.54 & 0.67 & 0.33 & 0.64 & 0.68 & 0.72 & 0.69 & 0.59 \\
\hline (6)' Europe-COF & 0.57 & 0.56 & 0.54 & 0.36 & 0.39 & 0.52 & 0.37 & 0.50 & 0.53 & 0.53 & 0.58 & 0.61 \\
\hline $\begin{array}{l}(7)^{\prime} \text { South America- } \\
\text { NCF }\end{array}$ & 0.59 & 0.65 & 0.72 & 0.52 & 0.52 & 0.65 & 0.61 & 0.73 & 0.53 & 0.42 & 0.80 & 0.73 \\
\hline $\begin{array}{l}(8)^{\prime} \text { South America - } \\
\text { CIF }\end{array}$ & 0.61 & 0.61 & 0.72 & 0.50 & 0.53 & 0.66 & 0.60 & 0.76 & 0.56 & 0.47 & 0.81 & 0.71 \\
\hline $\begin{array}{l}\mathbf{9})^{\prime} \text { South America- } \\
\text { COF }\end{array}$ & 0.73 & 0.60 & 0.56 & 0.50 & 0.61 & 0.56 & 0.61 & 0.64 & 0.66 & 0.53 & 0.74 & 0.62 \\
\hline $\begin{array}{l}\text { (10)' North America- } \\
\text { NCF }\end{array}$ & 0.56 & 0.48 & 0.50 & 0.59 & 0.44 & 0.39 & 0.54 & 0.39 & 0.30 & 0.32 & 0.55 & 0.56 \\
\hline $\begin{array}{l}\text { (11)' North America- } \\
\text { CIF }\end{array}$ & 0.53 & 0.52 & 0.52 & 0.39 & 0.50 & 0.49 & 0.42 & 0.46 & 0.46 & 0.46 & 0.54 & 0.51 \\
\hline $\begin{array}{l}\text { (12)' North America- } \\
\text { COF }\end{array}$ & 0.61 & 0.63 & 0.50 & 0.52 & 0.47 & 0.54 & 0.46 & 0.61 & 0.64 & 0.68 & 0.50 & 0.46 \\
\hline$(13)^{\prime}$ Africa-NCF & 0.61 & 0.61 & 0.72 & 0.19 & 0.60 & 0.72 & 0.46 & 0.64 & 0.75 & 0.62 & 0.74 & 0.62 \\
\hline$(\mathbf{1 4})^{\prime}$ Africa-CIF & 0.58 & 0.57 & 0.73 & 0.20 & 0.64 & 0.77 & 0.43 & 0.67 & 0.74 & 0.58 & 0.73 & 0.60 \\
\hline$(\mathbf{1 5})^{\prime}$ Africa-COF & 0.52 & 0.51 & 0.64 & 0.26 & 0.70 & 0.70 & 0.49 & 0.58 & 0.69 & 0.52 & 0.64 & 0.61 \\
\hline
\end{tabular}

Source: Calculated by the authors in MATLAB and EXCEL according to the selected capital flows data from the IMF Balance of Payments Statistics.

Note: The first row is 12 capital flows (net and gross) cycle series in four regions (Asia, Europe, North America and Oceania) of advanced economies, and the first

column is 15 capital flows (net and gross) cycle series in five regions (Asia, Europe, South America, North America and Africa) of emerging and developing economies. 


\section{CONCLUSION}

In this paper, we first investigated the cyclical characteristics of capital flows of 60 representative economies from the perspectives of duration, amplitude and speed using the BBQ algorithm. The main findings are as follows: small economies generally have greater amplitude and speed of net capital flows compared with large economies. For the three types of capital flows, the amplitude and speed of other investment and portfolio investment are significantly greater than that of direct investment in most economies. Furthermore, we also used the BBQ algorithm to identify and analyze the regional capital flows cycle. The common feature of most regions is that the duration of net capital flows cycle significantly shortened after the 2008 global financial crisis. However, in contrast to advanced countries, the amplitude of the net capital flows cycle in emerging and developing countries has enlarged significantly. Finally, we examined the regional synchronization of capital flows (net and gross) cycles. The results suggest that the degree of regional synchronization of capital flows cycles within advanced economies is high, while that within emerging and developing economies is relatively low. In addition, capital flows cycles between advanced Asian economies and any region of emerging and developing economies is highly synchronized.

This paper provides three policy insights. First, the higher the amplitude and speed of capital flows cycle, the more severe the strike on financial markets, especially at the contraction stage of the capital flows cycle. Therefore, risk prevention should be strengthened for economies with such characteristics and fragile financial systems (such as Malaysia and Thailand). Second, as capital flow volatility has increased significantly in most regions around the world since the 2008 global financial crisis, the relevant sectors should increase global portfolio diversification to cope with the risks caused by large fluctuations in capital flows. Third, there is high degree of synchronization of capital flows cycles among many regions of advanced, emerging and developing economies, especially between the advanced Asian regions and other regions. Such regional synchronization may work as a financial "gas pedal" to influence the global business cycles. Therefore, policy departments should focus on regional collaboration when facing the cyclical shocks of capital flows.

Funding: This work was supported by the Key Program of the National Natural Science Foundation of China [grant number: 71532013] and the Major Program of the National Social Science Foundation of China [grant number: 19ZDA062].

Competing Interests: The authors declare that they have no competing interests.

Acknowledgement: All authors contributed equally to the conception and design of the study.

\section{REFERENCES}

Baele, L., Bekaert, G., Inghelbrecht, K., \& Wei, M. (2020). Flights to safety. The Review of Financial Studies, 33(2), 689746.Available at: https://doi.org/10.1093/rfs/hhz055.

Bluedorn, M. J. C., Duttagupta, R., Guajardo, M. J., \& Topalova, P. (2013). Capital flows are fickle: Anytime, anywhere. IMF Working Paper No. 13/183. International Monetary Fund, Washington, D.C., USA.

Broner, F., Didier, T., Erce, A., \& Schmukler, S. L. (2013). Gross capital flows: Dynamics and crises. Journal of Monetary Economics, 6O(1), 113-133.

Broner, F., \& Rigobon, R. (2004). Why are capital flows so much more volatile in emerging than in developed countries? Working Papers Central Bank of Chile No.328. Central Bank of Chile, Santiago, Chile.

Broto, C., Díaz-Cassou, J., \& Erce, A. (2011). Measuring and explaining the volatility of capital flows to emerging countries. Journal of banking E Finance, 35(8), 1941-1953.Available at: https://doi.org/10.1016/j.jbankfin.201 1.01.004.

Burger, M. J., \& Ianchovichina, E. I. (2014). Surges and stops in FDI flows to developing countries: Does the mode of entry make a difference? Policy Research Working Paper No.6771. World Bank, Washington, D.C., USA.

Caballero, R. J., \& Krishnamurthy, A. (2008). Collective risk management in a flight to quality episode. The Journal of Finance, 63(5), 2195-2230 
Calderón, C., \& Kubota, M. (2014). Ride the wild surf: an investigation of the drivers of surges in capital inflows. World Bank Policy Research Working Paper No. 6753. World Bank, Washington, D.C., USA.

Calvo, G. A. (1998). Capital flows and capital-market crises: The simple economics of sudden stops. Journal of Applied Economics, 1(1), 35-54.Available at: https://doi.org/10.1080/15140326.1998.12040516.

Cowan, K., De Gregorio, J., Micco, A., \& Neilson, C. (2008). Financial diversification, sudden stops, and sudden starts. In K. Cowan, S. Edwards, \& R. O. Valdés (Eds.), Current account and external finance (pp. 159-194). Santiago, Chile: Central Bank of Chile.

Dagher, \& Jihad, C. (2010). Sudden stops, output drops, and credit collapses. IMF Working Paper No. 10/176. International Monetary Fund, Washington, D.C.

Davis, J. S. (2015). The cyclicality of (bilateral) capital inflows and outflows. Globalization and Monetary Policy Institute Working Paper No.247. Federal Reserve Bank of Dallas, Dallas, USA.

Forbes, K. J., \& Warnock, F. E. (2012). Capital flow waves: Surges, stops, flight, and retrenchment. Journal of International Economics, 88(2), 235-251.Available at: https://doi.org/10.1016/j.jinteco.2012.03.006.

Forbes, K. J., \& Warnock, F. E. (2020). Capital flow waves—or ripples? Extreme capital flow movements since the crisis. NBER Working Paper No. 26851. National Bureau of Economic Research, Cambridge, MA.

Giannetti, M., \& Laeven, L. (2012). The flight home effect: Evidence from the syndicated loan market during financial crises. Journal of Financial Economics, 104(1), 23-43.Available at: https://doi.org/10.1016/j.jfineco.2011.12.006.

Harding, D., \& Pagan, A. (2002). Dissecting the cycle: A methodological investigation. Journal of Monetary Economics, 49(2), 365381.Available at: https://doi.org/10.1016/S0304-3932(01)00108-8.

Harding, D., \& Pagan, A. (2006). Synchronization of cycles. Journal of Econometrics, 132(1), 59-79.Available at: https://doi.org/10.1016/j.jeconom.2005.01.023.

Kim, Y. J., \& Zhang, J. (2020). International capital flows: Private versus public flows in developing and developed countries. FRB of Chicago Working Paper No. 2020-27. Federal Reserve Bank of Chicago, Chicago.

Neumann, R. M., Penl, R., \& Tanku, A. (2009). Volatility of capital flows and financial liberalization: Do specific flows respond differently? International Review of Economics \& Finance, 18(3), 488-501.Available at: https://doi.org/10.1016/j.iref.2008.04.005.

Obstfeld, M. (2012). Financial flows, financial crises, and global imbalances. Journal of International Money and Finance, 31 (3), 469-480.Available at: https://doi.org/10.1016/j.jimonfin.2011.10.003.

Park, H. (2018). The cyclical properties of capital inflows in emerging market economies. International Review of Economics \& Finance, 56, 371-382.Available at: https://doi.org/10.1016/j.iref.2017.11.006.

Reinhardt, D., \& Dell'Erba, S. (2013). Not all capital waves are alike: A sector-level examination of surges in FDI inflows. Bank of England Working Paper No. 474. Bank of England, London, U.K.

Reinhart, C. M., \& Reinhart, V. R. (2008). Capital flow bonanzas: An encompassing view of the past and present. NBER Working Paper No. 1432 1. National Bureau of Economic Research, Cambridge, MA.

Rey, H. (2015). Dilemma not trilemma: The global financial cycle and monetary policy independence. NBER Working Paper No. 21162. National Bureau of Economic Research, Cambridge, MA.

Rothenberg, A. D., \& Warnock, F. E. (2011). Sudden flight and true sudden stops. Review of International Economics, 19(3), 509524.Available at: https://doi.org/10.1111/j.1467-9396.2011.00962.x.

Schmidt, T., \& Zwick, L. (2015). Uncertainty and episodes of extreme capital flows in the Euro Area. Economic Modelling, 48, 343-356.Available at: https://doi.org/10.1016/j.econmod.2014.10.047.

Vayanos, D. (2004). Flight to quality, flight to liquidity, and the pricing of risk. NBER Working Paper No. 10327. National Bureau of Economic Research, Cambridge, MA.

Views and opinions expressed in this article are the views and opinions of the author(s), Asian Economic and Financial Review shall not be responsible or answerable for any loss, damage or liability etc. caused in relation to/arising out of the use of the content. 